\title{
Chronic Inflammatory Bowel Disease (CIBD) and Celiac disease, are these linked in the horse?
}

\author{
René van den Hoven', Denise Thaller ${ }^{2}$ and Peter Bankuti? \\ ${ }^{1}$ Clinical Department of Small Animals and Horses, Section Equine Internal Medicine \\ 2 Department for Pathobiology, Institute of Pathology and Forensic Veterinary Medicine, Vetmeduni Vienna, Austria.
}

\begin{abstract}
Summary: Chronic inflammatory bowel disease (CIBD) is a disorder of the mucosa and submucosa characterized by infiltration of inflammatory cells. Diagnosis of the various forms is based on the degree of inflammation and the predominant type of infiltrating leucocytes. The cause of this abnormal cellular infiltrate has been linked to abnormal immune responses to bacterial, viral, parasitic, or dietary antigens. Celiac disease $(C D)$ is a chronic inflammatory disease induced in genetically susceptible people by the ingestion of the irritant gluten and possibly modulated by other environmental cofactors. An abnormal immune response against gluten is the trigger for development of CD. CD is characterized by a large heterogeneity of clinical signs ranging from asymptomatic to severely symptomatic. Chemokine receptors play a fundamental role in leukocyte migration and one of the important chemokine receptors is CXCR3, which is expressed abundantly on human T-cells, and particularly on those T-cells associated with activation and inflammation. CXCR3 seems to play a role in equine immune response as well (Johnstone et al. 2016). Gliadins, the major gluten protein types, release zonulin after coupling to CXCR3. This subsequently results in increased permeability of the small intestinal mucosa and concurrent inflammation. A potential role of gluten in equine chronic inflammatory bowel disease was recently reported. The current pilot study aimed to show the expression levels of the CXCR3 receptor in small intestinal samples of healthy horses and horses histologically diagnosed with any of the disorders belonging to the CIBD syndrome. The electronic patient files of the University Equine Hospital of the Vetmeduni Vienna were searched for cases with protein-losing enteropathy, weight loss, chronic enteritis and performed oral glucose tolerance tests. Furthermore, only cases from which intestinal samples had been kept in the repository were selected. Freshly collected small intestinal samples from 8 randomly chosen slaughter horses without any overt clinical signs were collected and used as control. Small bowel tissue was routinely stained with hematoxylin and eosin (HE) and the presence of CXCR3 in cells was shown by an in-situ immunofluorescence technique. Stained sections were analyzed using an Imager microscope and immunofluorescence of each individual slide was expressed as mean fluorescence intensity $\left(\mathrm{MFI} / \mu \mathrm{m}^{2}\right) \mathrm{using}$ specific software. A total of 160 files with clinical histories of intestinal malabsorption, chronic weight loss or small intestinal inflammation were selected and further analyzed. Finally, only 7 cases fulfilled all criteria of equine CIBD. One of the cases had a concurrent adenocarcinoma of the caecum. One case showed histological signs of proliferative enteritis suggestive for infection with Lawsonia intracellularis, but the organism could no longer be detected in the sections. MFI was not significantly different between the diseased and the control group ( $p=0.613$ ). One control horse reacted unexpectedly strong and was considered as unhealthy. Therefore, it was excluded from the control group. This pilot study showed that small intestinal mucosae of horses with histological lesions suggestive for CIBD did not express significantly more CXCR3 on cells in the gut walls than did randomly chosen slaughter horses. Therefore, this study could not prove that upregulation of CXCR3 on mucosal cells, possibly lymphocytes, is a specific response to gluten. Gluten allergy in the horse may be mediated by other cytokine receptors as well. Whether gluten allergy is the major cause of CIBD in horses cannot yet be concluded.
\end{abstract}

Keywords: horse, celiac diseases, inflammatory bowel disease, CXCR3 immunohistochemistry.

Citation: van den Hoven R., Thaller D., Bankuti P. (2016) Chronic Inflammatory Bowel Disease (CIBD) and Celiac Disease, are these linked in the horse? Pferdeheilkunde 33, 23-29; DOI 10.21836/PEM20170103

Correspondence: Prof. René van den Hoven, Veterinary Universitiy of Vienna, Clinical Department of Small Animals and Horses, Section Equine Internal Medicine, Veterinärplatz 1,1210 Vienna, Austria; e-mail: rene.vandenhoven@vu-wien.ac.at

\section{Introduction}

The differential diagnosis for weight loss, dependent edema, lethargy or diarrhea with or without recurrent signs of mild colic in the horse should include chronic inflammatory bowel disease (CIBD). A condition strongly suggestive for CIBD is the presence of protein-losing enteropathy (Schumacher et al. 2000). Although hypoproteinemia due to enteral protein loss is a major aspect of many cases of CIBD, maldigestion and malabsorption are other concurrent factors leading or contributing to hypoproteinemia. The cause of malabsorption and maldigestion of nutrients is mostly due to lesions in the small intestine (Kalck 2009). Occasionally, in advanced disease, the large intestine is also involved (Kalck 2009).

Enteral protein loss is difficult to prove directly. Indirect evidence is often obtained by measuring the absorption of orally administered glucose (oral glucose tolerance test, OGTT) or xylose (Roberts and Hill 1973, Roberts and Norman 1979). The basic idea behind this test is that gut inflammation will also involve enterocyte function and therefore poor absorption of amino acids next to leaking of protein likely will be present. CIBD is recognized as an infiltration of the mucosa and submucosa with inflammatory cells (Mair et al. 2006, Schumacher et al. 2000). The categorization of the various forms of CIBD is based on the degree of inflammation and the predominant type of infiltrating leucocytes (Divers et al., 2006).

The cause of the abnormal cellular infiltrate is associated with abnormal immune responses to bacterial, viral, parasitic or dietary antigens (Schumacher et al 2000). The equine CIBD syndrome includes granulomatous enteritis $(\mathrm{GE})$, multisystemic eosinophilic epitheliotropic disease (MEED), lymphocyticplasmacytic enterocolitis (LPE), and idiopathic eosinophilic enterocolitis (EC) (Schumacher et al. 2000). 
Cimprich (1974) noted the histologic similarity between equine GE and Crohn's disease of man. The cause of MEED is speculated to be related to episodes of immediate type hypersensitivity reactions, likely evoked by dietary, inhaled, or parasitic antigens (Schumacher et al. 2000). LPE is characterized by a moderate to severe infiltration of lymphocytes and/or plasma cells, accompanied by morphological abnormalities, such as varying degrees of villus blunting, fusion or atrophy and the presence of mucosal and/or submucosal edema (Kemper et al. 2000). These changes in LPE share similarities with celiac disease of man.

Celiac disease (CD) is a chronic inflammatory disease characterized by flattened villi of the small bowel mucosa, and is induced in genetically susceptible people by the ingestion of irritant gluten and possibly other environmental cofactors. An abnormal immune response against gluten is the cause of CD (Di Sabatino and Corazza 2009). Gluten proteins are present in wheat, rye, and barley. Gluten proteins have a complex chemistry and are responsible for the baking properties of wheat (Wieser 2007). The gliadins are a group of major gluten proteins from which the immunogenicity and toxicity of several epitopes has been established (Kagnoff 2007).

CD is characterized by a diverse clinical heterogeneity ranging from asymptomatic to severely symptomatic. Clear clinical signs with frank malabsorption and an increased morbidity are attributable to the underlying immune disorder (Di Sabatino and Corazza 2009). The prevalence of CD is serologically tested using $\lg A$ antigliadin antibodies. Positive responders are subsequently tested for the presence of antiendomysial antibodies and IgA antihuman tissue transglutaminase (hTTG) antibodies (Di Sabatino and Corazza 2009). These tests enable screening of large populations and select only those people who should undergo a gut biopsy to confirm CD histologically (Di Sabatino and Corazza 2009).

According to the Kikkuli Text from 1400 BC, Assyrian horses were fed mixtures of wheat and barley next to oats, whereas in Europe about 1000 years later, horses were mainly kept on grass and if supplemented with cereals, then with oats, the cheaper choice. Modern horse feeding practices include feeding of pellets and mueslis. Both may contain significant amounts of wheat, barley or rye. Since the demand of glutenfree food for man has strongly increased, extracted gluten has found its way to the animal feed industry. The change from oat to mueslis and pellets in the daily rations of horses could result in the occurrence of an equine form of CD. Van den Kolk et al. (2012) investigated the potential role of gluten in CIBD. Two CIBD affected horses, as well as a horses fed a gluten-rich diet had significantly higher $\lg A$ antibodies to human recombinant and guinea pig tissue transglutaminase (TGA) than horses on a gluten poor diet. However, the authors could not show that the CIBD group had higher levels of any of the CD related antibodies compared to the healthy gluten rich fed control group. Nevertheless, one case of CIBD with markedly increased TGA, EMA and DGPA levels was identified in that study. After 6 months of feeding a gluten-free diet, this horse showed decreased its antibody levels and showed an amelioration of duodenal histopathologic lesions (van den Kolk at al. 2012).

Chemokine receptors play a fundamental role for leukocyte migration and one of the important chemokine receptors is CXCR3, which is expressed abundantly on human T-cells, and particularly on those T-cells associated with activation and inflammation (Qin et al. 1998). Meanwhile it is known that CXCR3 is the target for gliadin and the CXCR3-Gliadin coupling releases zonulin (Lammers et al. 2008). Zonulin causes increased small intestinal mucosal permeability (Clemente et al. 2003) and subsequent inflammation. The current pilot study aimed to show the expression levels of the CXC3 receptor in jejunal samples of healthy horses and horses histologically diagnosed with any of the disorders belonging to the CIBD syndrome.

\section{Material and Methods Horses}

The Vienna Equine Hospital's electronic files were searched for the entries protein-losing enteropathy, weight loss, chronic enteritis and performed oral glucose tolerance tests. One

\begin{tabular}{|c|c|c|c|c|c|c|c|}
\hline Table I & Horses sa & led in the study. & me (Nrs. & introl slaughte & $\operatorname{ses}(8-15)$ & manifest; & mptom not \\
\hline $\mathrm{Nr}$. & Age (years) & Breed & Gender & Weight loss & Diarrhoea & Apathy & Colic \\
\hline 1 & 18 & Haflinger & Mare & + & - & - & + \\
\hline 2 & 21 & Standardbred & Mare & + & - & - & + \\
\hline 3 & 11 & Riding pony & Gelding & - & - & - & + \\
\hline 4 & 1 & Warmblood & Stallion & + & - & + & - \\
\hline 5 & 16 & Arab & Mare & - & - & + & - \\
\hline 6 & 16 & Warmblood & Gelding & + & + & --- & + \\
\hline 7 & 9 & Warmblood & Gelding & + & - & - & - \\
\hline 8 & 20 & Warmblood & Gelding & - & - & ND & ND \\
\hline 9 & 20 & Warmblood & gelding & - & - & ND & ND \\
\hline 10 & 17 & Warmblood & Gelding & - & - & ND & ND \\
\hline 11 & 12 & Warmblood & Mare & - & - & ND & ND \\
\hline 12 & 16 & Warmblood & Mare & - & - & ND & ND \\
\hline 13 & 12 & Warmblood & Mare & - & - & ND & ND \\
\hline 14 & 18 & Warmblood & Mare & - & - & ND & ND \\
\hline 15 & 19 & Warmblood & Stallion & - & - & ND & ND \\
\hline
\end{tabular}


hundred sixty files were selected for further evaluation and were analyzed for histologically confirmed changes in the gut that were consistent with CIBD, like GE, MEED, LPE and EC. After rigorous scrutinizing, only 7 cases with suitable clinical histories and partly complete laboratory results were selected. From these cases, archived paraffin embedded material was available for further processing. Furthermore, freshly collected jejunal samples from 8 randomly chosen slaughter horses without any overt clinical signs were collected, fixed in formalin, embedded in paraffin and routinely stained with hematoxylin and eosin $(\mathrm{HE})$, serving as control group.

\section{CXCR3 Staining}

The presence of CXCR3 on cells was shown by an in-situ immunofluorescence technique. Anti- equine-CXCR3 antibodies are not commercially available, but the amino acid sequence of human CXCR3 is $91 \%$ identical and has a further $7 \%$ similarity of the sequences of equine CXCR3 (www.uniprot.org). Therefore, it was considered very likely that a specific reaction of human CXCR3-Antibodies against equine CXCR 3 would occur. Three $\mu \mathrm{m}$ thick sections were prepared for receptor staining. Sections were de-paraffinized, rehydrated and heated in citrate buffer at $100^{\circ} \mathrm{C}$ for 20 minutes and at $\mathrm{pH}$ 6. Sections were treated with $3 \%$ donkey serum for blocking. Thereafter sections were incubated with a 1:200 diluted primary mouse anti-man antibody solution (MAB 160 100, Novus Biologicals, Abingdon, United Kingdom) overnight at $4{ }^{\circ} \mathrm{C}$ against human CXCR3. Subsequently, samples were incubated with a 1:500 dilution of IF marked antibodies (Invitrogen A-31571, Fisher Scientific, Vienna, Austria) for 60 minutes. For better identification of structures, cell nuclei were stained with diamidino-2-phenylindole (DAPI, Fisher Scientific, Vienna, Austria) for 10 minutes and cell proteins with Green Fluorescent Protein (GFP, Fisher Scientific, Vienna, Austria). Sections of human spleen as well as monkey tonsils, small bowel and lymphocytes were used as positive control to check CXCR3 activity of the primary antibodies. For specific activity against equine cells, equine spleen was used. MAB 160-100 stained sections were compared to negative control sections not incubated with MAB160-100.

\section{Histology}

A pathohistological examination of all HE stained samples of the control and the study group was performed.

Since it was difficult to quantify red light intensity of the samples with the un aided eye, therefore, the MAB-160 stained sections were analysed using an Axio Imager Z2 microscope (Carl Zeiss Microscopy GmbH, Jena, Germany). The microscope had multiple, varied built-in light filters. For the better detection of CXCR3, these filters were set at 625-655nm (excitation) and $665-715 \mathrm{~nm}$ (emission), for GFP the filter setting was $450-490 \mathrm{~nm}$ (excitation), 500-550 nm (emission) and for DAPI settings were $335-383 \mathrm{~nm}$ (excitation) and 420-470 nm (emission), respectively. Composite pictures were made with an Axiocam MRm Rev 3.1 Camera (Carl Zeiss Microscopy GmbH, Jena, Germany) with Software ZEN 2012 Pro (Carl Zeiss Microscopy GmbH, Jena, Germany). All sections were evaluated and measured at a magnification of $400 \mathrm{X}$. Using Fiji (Version 1.51 a) software, the immunofluorescence of the red channel was measured for each individual picture and expressed as mean fluorescence intensity $\left(\mathrm{MFI} / \mu \mathrm{m}^{2}\right.$.

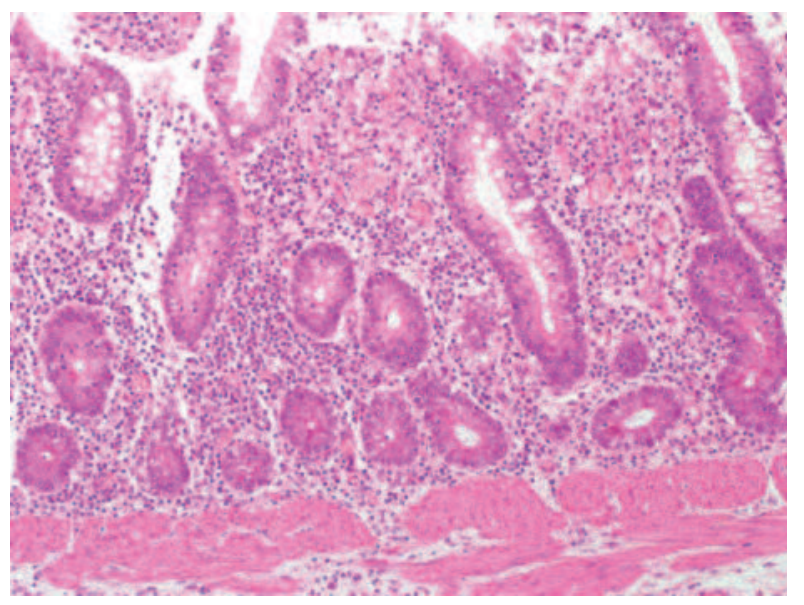

Fig. 1 Small intestine showing a mainly lymphocytic-plasmacytic infiltration; $\mathrm{HE} ; \times 200$.

\begin{tabular}{|c|c|c|c|c|c|c|c|}
\hline \multicolumn{8}{|c|}{ Available results of haematology and clinical pathology of CIBD diagnosed horses Nrs. 1 through 7.} \\
\hline Parameter (Reference interval & 1 & 2 & 3 & 4 & 5 & 6 & 7 \\
\hline$P C V \quad L / L \quad(0.32-0.55)$ & & & 0.30 & 0,26 & 0.38 & 0.55 & 0.33 \\
\hline WBC $\quad 10^{9} / \mathrm{L} \quad(5.0-10.0)$ & & & 18 & 10.8 & 6.0 & & 4.8 \\
\hline Tot Protein g/L $\quad(55-75)$ & 45 & 57 & 63 & & 74 & 52 & 49 \\
\hline Albumin $\mathrm{g} / \mathrm{L} \quad(24-45)$ & 12 & 25 & 27 & 15 & 34 & & 25 \\
\hline$\alpha$-globulin g/L $\quad(5-16)$ & 8 & & & 6.1 & & & \\
\hline B-globulin g/L (13-28) & 22 & & & 13 & & & \\
\hline Y-globulin g/L (4-9) & 5 & & & 9 & & & \\
\hline Fibrinogen $\mathrm{g} / \mathrm{L} \quad(1-4)$ & 1.1 & & & 2.5 & 1.3 & & 3.5 \\
\hline$(<13 I U / L)$ & 34 & & & 3 & 3 & & 46 \\
\hline$(<30 \mathrm{IU} / \mathrm{L})$ & 37 & & & 12 & 17 & & 16 \\
\hline$(<240 \mathrm{IU} / \mathrm{L})$ & 351 & & & & & & 294 \\
\hline $\mathrm{SAA} \mathrm{mg} / \mathrm{L} \mathrm{mg} / \mathrm{l} \quad(<10)$ & 0.4 & & & & & & \\
\hline Amylase $\quad(3-36 \mathrm{IU} / \mathrm{L})$ & 2 & & & & & & \\
\hline Creatinine $\mu \mathrm{mol} / \mathrm{I} \quad(<177)$ & & 97 & 124 & 106 & 106 & & 97 \\
\hline
\end{tabular}



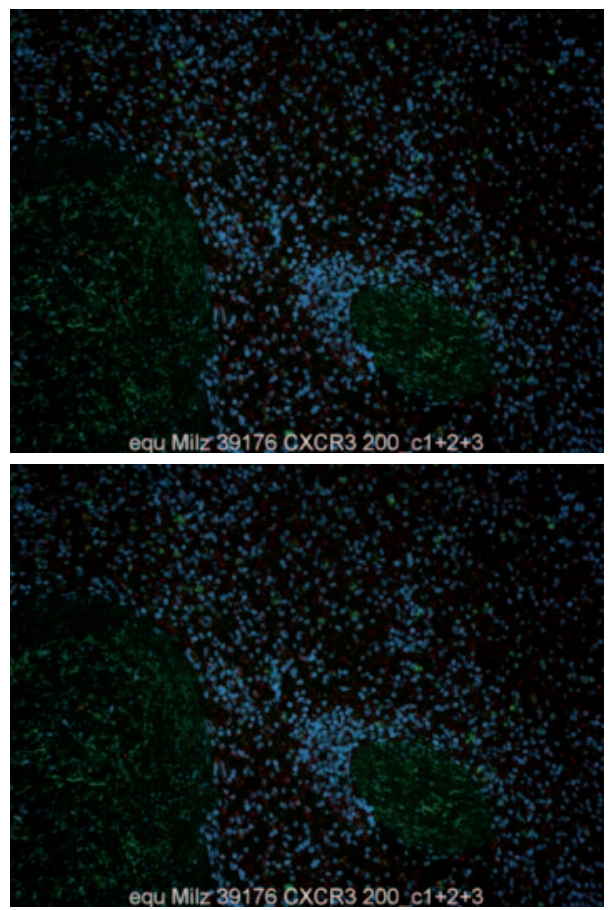

Fig.2 Quality and specificity control of Novus MAB-160 using equine spleen. Above: stained with MAB160, below: no MAB 160. Red stained is CXCR3; cellular protein is green by Green Fluorescent Protein (GFP) stain and blue stained by diamidino-2-phenylindole (DAPI) are the cell nuclei. The pictures show the difficulty to judge the staining intensity by the unaided eye even for a densely active tissue as the equine spleen.

\section{Results}

Retrospectively, only 7 cases with stored gut tissue fulfilled the criteria of equine CIBD according to their records. One case had a concurrent adenocarcinoma of the caecum. One other case (Nr. 4) showed histological signs of proliferative enteritis
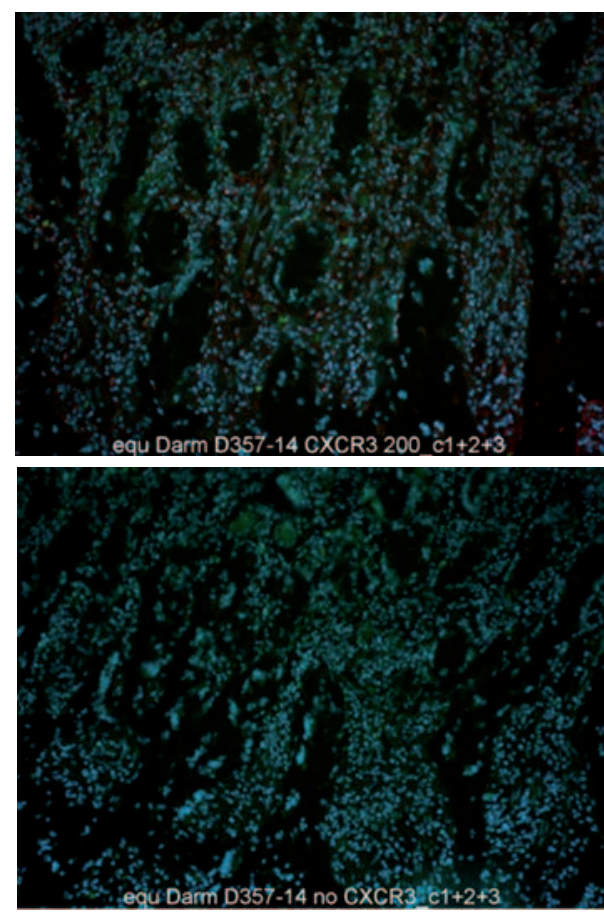

Fig 3 Above: staining of CXCR3 on cells in equine jejunal mucosa; below non CXCR stained control section..

suggestive for infection with Lawsonia intracellularis, but the organism could not be detected in the gut sections. This animal had anti Lawsonia antibody titre of 1:30 and there had been confirmed cases on the farm. The final selected study population and its clinical and pathologic characteristics are summarised in Tables 1-3. Clinical symptoms varied considerably and hypoproteinemia was not present in all cases (Table 2). Fig. 1 shows a jejunal section of an animal with severe lymphocytic-plasmacytic mucosal infiltration. In Fig 2 the positive staining of CXCR3 of what are likely mostly lymphocytes in spleen section is shown. In Fig 3 the staining of

Table 3 Horses sampled in the study. CIBD Syndrome (nrs. 1-7) and control slaughter horses (8-15). Main pathological lesions and mean CXCR3 colouring intensity $(\mathrm{MFI}) / \mu \mathrm{m}^{2}$ of sections.

\begin{tabular}{|c|c|c|}
\hline Nr. & Main gross pathologic and microscopic lesions & MFI (pro $\left.\mu \mathrm{m}^{2}\right)$ \\
\hline 1 & Small intestinal lymphocytic-plasmocytic enteritis and hypertrophic muscularis mucosae. Liver cirrhosis & 248 \\
\hline 2 & Hypertrophic ileum, chronic enteritis, local adenocarcinoma in caecum with hepatic metastases & 438 \\
\hline 3 & Chronic lymphoproliferative enteritis. focal diphteroid-necrotic lesions in jejunum, chronic typhlo-colitis & 479 \\
\hline 4 & $\begin{array}{l}\text { Chronic proliferative enteritis with increased numbers of fibroblasts and fibrocytes. No Lawsonia intracellularis organisms } \\
\text { found. }\end{array}$ & 218 \\
\hline 5 & Adipositas, gastritis, lymphocytic-plasmocytic entero-colitis & 334 \\
\hline 6 & Chronic duodenitis and thyflo-colitis, degeneration of myenteric plexus & 312 \\
\hline 7 & $\begin{array}{l}\text { Purulent pancreatitis, moderate enteritis with hypertrophy and lymphocytic plasmocytic infiltration of lamina propria, } \\
\text { diphtheric colitis }\end{array}$ & 301 \\
\hline 8 & No gross abnormalities, selected jejunum: normal histopathologic appearance & 13 \\
\hline 9 & No gross abnormalities, selected jejunum: normal histopathologic appearance & 474 \\
\hline 10 & No gross abnormalities, selected jejunum: normal histopathologic appearance & 3 \\
\hline 11 & No gross abnormalities, selected jejunum: normal histopathologic appearance & 436 \\
\hline 12 & No gross abnormalities, selected jejunum: normal histopathologic appearance & 401 \\
\hline 13 & No gross abnormalities, selected jejunum: normal histopathologic appearance & 495 \\
\hline 14 & No gross abnormalities, selected jejunum: normal histopathologic appearance & 184 \\
\hline 15 & No gross abnormalities, selected jejunum: normal histopathologic appearance & 147 \\
\hline
\end{tabular}


CXCR3 on cells, likely lymphocytes, in the equine jejunal mucosa is shown.

Boxplots are given in Fig 4 for mean fluoroscopic intensity of the red channel $\left(\mathrm{MFI} / \mu \mathrm{m}^{2}\right)$. None of these parameters were significantly different between the diseased and the control group $(p=0.613)$, even after exclusion of horse Nr.4. One control horse reacted unexpectedly strong and was considered as unhealthy. Therefore, it was excluded from the control group.

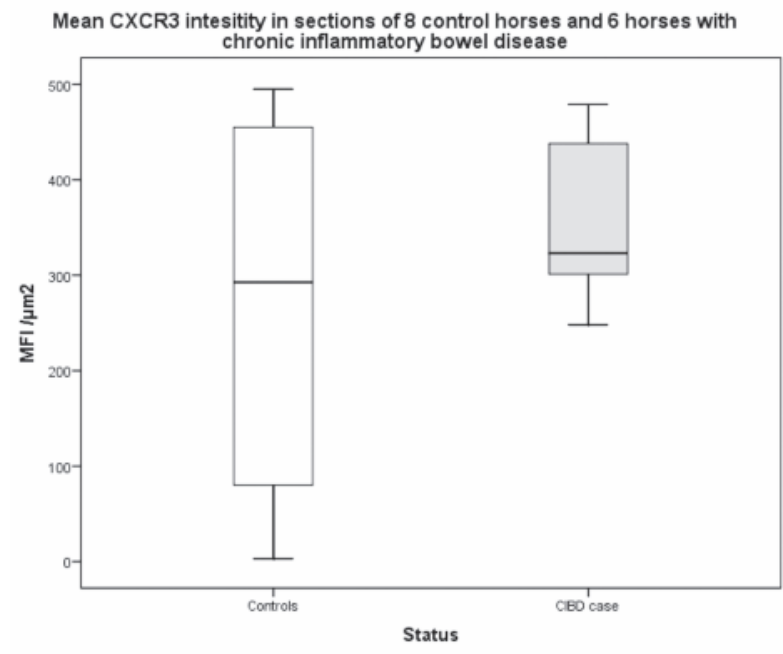

Fig. 4 Comparison of CXCR3 red staining intensity (MFI/ $\mu \mathrm{m} 2$ ) between 8 control horses and 6 diseased horses. The data of the yearling with suspected post Lawsonia intercellularis infection were excluded.

\section{Discussion}

A suitable selection of sufficient tissues from CIBD cases appeared more difficult than expected. Many horses diagnosed with CIBD were sent home with treatment protocols and got lost for follow up. The few samples from cases that fulfilled the criteria of CIBD came from horses with variable clinical histories and signs. About half of these horses actually showed weight loss, whereas the other suffered from acute or chronic colic. For a reliable clinical diagnosis of CIBD, a thoroughly conducted panel of tests should be carried out. However, this was mostly not done, mainly due to financial restraints. Therefore, it is likely that CIBD is easily over diagnosed although it is rather uncommon in our clinic population.

In our hospital population lymphocytic-plasmacytic enterocolitis (LPE), a form of CIBD syndrome (Schumacher et al. 2000) was often tentatively diagnosed based on histopathologic evaluation of rectal or duodenal biopsies. The diagnostic tests helpful in establishing a tentative diagnosis of LPE include rectal mucosal biopsies and an OGTT (Kemper et al. 2000), however, this was not performed in most of our suspected cases.

This study showed that if rigid criteria are applied for re evaluation of CIBD diagnosed cases, only few cases could be definitely marked as CIBD. In the majority of our reviewed cases, low plasma albumin levels, abnormal OGTT and the pathological alterations in intestinal samples from euthanized horses or in duodenal or rectal biopsies often did not support the diagnosis of CIBD. Merely the finding of mild to moderate thickening of the small intestinal wall at ultrasonography was often the basis of many tentative diagnoses of CIBD. Supportive evidence for CIBD was further sought in about one third of the cases by histological evaluation of trans endoscopically taken duodenal biopsies or by rectal biopsies. However, in relatively few horses variably increased numbers of lymphocytes and plasma cells were infiltrating the mucosa and submucosa. The interpretation of this finding remains ambiguous as Kemper et al. (2000) consider that just a mild infiltration of lymphocytes and plasma cells in the lamina propria of the gut, or the presence of inflammatory infiltrates characterized as primarily neutrophilic, eosinophilic or histiocytic, are not supportive for the diagnosis of LPE. LPE was limited to the small intestine in only $36 \%$ of horses in the study by Kemper et al. (2000), while both small and large intestine were affected in $64 \%$ of horses. Elevated $\lg A$ concentration in 4 horses in that study indicate the potential role of an immune response.

Recently more evidence was produced that special immune responses could play a role in human IBD. Olofsson et al. (2015) suggest that Th17 cells are involved in active IBD, possibly through recruitment of neutrophils via IL-17A, in combination with inadequate suppression of the inflammatory response by regulatory T-cells.

Although the group median $\mathrm{MFI} / \mu \mathrm{m}^{2}$ in the selected cases was slightly higher than in the controls (Fig. 4), this study could not support that a strong CXCR3 up-regulation was clearly present in all CIBD cases. Therefore, assuming that if gluten intolerance in horses exists and CXCR3 in equine lymphocytes will be strongly upregulated as in man, the results of our study suggests that CD is not a major cause of equine CIBD. However, lymphocyte characteristics must be further studied and more samples from well diagnosed and documented cases need to be analyzed before a final judgement can be made. Interestingly, a past infection with Lawsonia intracellularis was not associated with a strong CXCR3 upregulation.

Since there are more forms of wheat allergies in man than CD (Uhde et al. 2016), similar conditions may be present in the horse. These forms need to be studied in more detail. Patients suffering these forms of wheat allergy experience a range of symptoms in response to ingestion of wheat and related cereals, yet they lack the characteristic serological, histological or genetic markers of celiac disease

In conclusion, this study did not support the likelihood that $C D$ in horses is a major cause of CD, but it cannot be excluded that wheat allergies in horses exist which may be mediated via different receptors than CXCR3.

\section{References}

Clemente M. G., Virgiliis S. D, Kang J. S., Macatagney R., Musu M. P., Pierro M. R., Di Drago S., Congia M., Fasano A. (2003) Early effects of gliadin on enterocyte intracellular signaling involved in intestinal barrier function. Gut, 52, 218-223

Cimprich R. E. (1974) Equine granulomatous enteritis. Vet. Pathol. $11,535-547$

Di Sabatino A., Corazza G. R. (2009) Coeliac disease. Lancet 2009, 373, 1480-1493 
Divers T, Pelligrini Masini A., McDonough S. (2006) Diagnosis of inflammatory bowel disease in a Hackney pony by gastroduodenal endoscopy and biopsy and successful treatment with corticosteroids. Equine Vet. Educ. 18, 368-371

Hunter J. O. (2009) Do horses suffer from irritable bowel syndrome? Equine Vet. J. 41, 836-840

Johnstone S., Barsova J, Campos I, Frampton A. R. (2016) Equine herpesvirus type 1 modulates inflammatory host immune response genes in equine endothelial cells. Vet. Microbiol. 192, 52-59

Kagnoff M. F. (2007) Celiac disease: pathogenesis of a model immunogenetic disease. J. Clin. Invest. 117, 41-49

Kalck K. (2009) Inflammatory Bowel Disease in Horses. Vet. Clin. Equine 25, 303-315

Lammers K. M., Lu R., Brownley J., Lu B., Gerard C., Thomas K., Rallabhandi P., Shea Donohue T., Tamiz A., Alkan S., Netzel-Arnett S., Antalis T., Vogel S. N., Fasano A. (2008) Gliadin induces an increase in intestinal permeability and zonulin release by binding to the chemokine receptor CXCR3. Gastroenterology 135,194-204

Lindberg R., Nygren A., Persson S. G. B. (1996) Rectal biopsy diagnosis in horses with clinical signs of intestinal disorders: a retrospective study of 116 cases. Equine vet. J. 28, 275-284

Mair T., Pearson G. R., Divers T. J. (2006) Malabsorption syndromes in the horse. Equine Vet. Educ. 18, 383-392

Olofsson K. M., Hiertner B., Fossum C., Charles M., Press C. M., Lindberg R. (2015) Expression of T helper type 17 (Th17)-associa- ted cytokines and toll like receptor 4 and their correlation with Foxp3 positive cells in rectal biopsies of horses with clinical signs of inflammatory bowel disease. Vet. J. 206,97-104

Roberts. M. C., Hill F. W. G. (1973) The oral glucose tolerance test in the horse. Equine Vet. J. 5, 171-173

Roberts M. C., Norman P. (1979) A re-evaluation of the $\mathrm{d}(+)$ xylose absorption test in the horse. Equine vet. J. 11, 239-243

Qin S., Rottman J. B., Myers P., Kassam N., Weinblatt M., Loetscher M., Koch A. E., Moser B., Mackay C. R. (1998) The Chemokine Receptors CXCR3 and CCR5 Mark Subsets of T Cells Associated with Certain Inflammatory Reactions J. Clin. Invest. 101, 746-754

Schumacher J., Edwards J. F., Cohen N. D. (2000) Chronic idiopathic inflammatory bowel diseases of the horse. J. Vet. Intern. Med. $14,258-365$

Uhde M, Ajamian M, Caio G, De Giorgio R., Indart A., Green P. H., Verna E. C., Volta U., Alaedini A. (2016) Intestinal cell damage and systemic immune activation in individuals reporting sensitivity to wheat in the absence of coeliac disease. Gut 25; doi:10.1136/gutinl 2016311964

van der Kolk J. H., van Putten L. A., Mulder C. J., Grinwis G. C. M., Reijim M., Butler C. M., von Blomberg B. M. E. (2012) Gluten dependent antibodies in horses with inflammatory small bowel disease (ISBD). Vet. Quar. 32, 3-1 1

Wieser H. (2007) Chemistry of gluten proteins. Food Microbiol. 24,1 15-119 